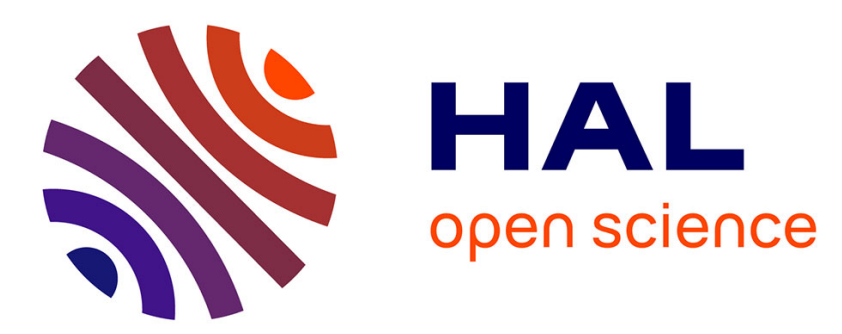

\title{
DermaDL: Advanced Convolutional Neural Networks for Automated Melanoma Detection
}

\author{
Jose Rodrigues, Bruno Brandoli, Sihem Amer-Yahia
}

\section{To cite this version:}

Jose Rodrigues, Bruno Brandoli, Sihem Amer-Yahia. DermaDL: Advanced Convolutional Neural Networks for Automated Melanoma Detection. 2020 IEEE 33rd International Symposium on Computer-Based Medical Systems (CBMS), Jul 2020, Rochester, France. pp.504-509, 10.1109/CBMS49503.2020.00101 . hal-02972535

\section{HAL Id: hal-02972535 \\ https://hal.science/hal-02972535}

Submitted on 12 Nov 2020

HAL is a multi-disciplinary open access archive for the deposit and dissemination of scientific research documents, whether they are published or not. The documents may come from teaching and research institutions in France or abroad, or from public or private research centers.
L'archive ouverte pluridisciplinaire HAL, est destinée au dépôt et à la diffusion de documents scientifiques de niveau recherche, publiés ou non, émanant des établissements d'enseignement et de recherche français ou étrangers, des laboratoires publics ou privés. 


\title{
DermaDL: Deep Learning for Early Melanoma Detection
}

\author{
Daniel M. de Lima, Sihem Amer-Yahia, Jose F Rodrigues-Jr \\ University of Sao Paulo - Sao Carlos, SP, Brazil \\ CNRS, Univ. Grenoble Alpes - Grenoble-Alpes, France
}

\begin{abstract}
In this paper we use state-of-the-art deep convolutional neural networks for computer-aided melanoma detection. As a result, we present the DermaDL mobile application, where dermatologists can use neural network modules for automated lesion analysis with the aim of identifying and classifying skin lesions with regard to malignancy. The proposed methodology includes a preprocessing step for data organization, normalization, augmentation and image segmentation; after that, we will employ transfer learning from state-of-the-art models previously elaborated for the ImageNet Large Scale Visual Recognition Challenge (ILSVRC), using extensively trained Inception-type neural network models. Finally, the models will be optimized for mobile processors, allowing for mobility and convenient use. This method can classify several types of skin lesions present in the International Skin Imaging Collaboration (ISIC) archive with at least $90 \%$ accuracy, purposefully documenting and triaging clinical cases before further thorough examination.

Index Terms - deep learning; dermatology; dermoscopy; early detection; melanoma; mobile; skin cancer
\end{abstract}

\section{INTRODUCTION}

In the last decade, deep CNNs (convolutional neural networks) have achieved unprecedented levels of performance due to two factors: the explosion in data production, and the popularization of high-density parallel hardware in the form of general-purpose computing on graphic processor units (GPGPU). The GPGPU computing approach made it possible to build neural networks with novel architectures and dozens of layers suited for intricate tasks, such as image, video and speech recognition, achieving precision near or surpassing classical multi-layer perceptions (MLP), other machine learning algorithms, and even human analysts in certain data domains, such as the ILSVRC (ImageNet Large Scale Visual Recognition Challenge) - coining the term deep learning [1].

Deep learning (DL) can be directed towards digital skin imaging to recognize disease patterns such as melanoma. $\mathrm{Cu}$ taneous melanoma is a class of cancerous diseases in the skin which becomes life-threatening when the metastatic (advanced spread) stage begins, causing more than 50 thousand deaths annually worldwide [2]. This disease is promptly curable if recognized and treated in its initial stages, with previous reports in the US of $93 \%$ of patients surviving 15+ years when their lesions are $<1 \mathrm{~mm}$, but just $42 \%$ when their lesions grow more than $4 \mathrm{~mm}$ [3]. Digital images of skin lesions have potential for educating the general public and support health providers in early detection, as well as directly supporting the diagnosis through teledermatology, clinical decision support, and automated risk assessment.
This recent development of DL opens up the possibility to power a plethora of complex recognition applications, e.g. for healthcare [4], forensic sciences [5], manufacturing quality control [6], agriculture [7], and many others. One of such, a study by Esteva et al. [8] has previously demonstrated the ability to classify cancerous skin lesions by retraining the Inception-v3 CNN [9] over a carefully prepared dataset (with more than 120k images) from open-access and closedaccess dermatology archives of several Institutions and Clinical Hospitals - one of these datasets was the ISIC (International Skin Imaging Collaboration) Archive, also used in our method. In that study, the methodology is evaluated by the Specificity/Sensitivity AUC metric, and achieves a classification accuracy similar to that of board-certified dermatologists.

In this context, this paper follows up the current DL research by presenting a novel application of image recognition for clinical decision support at the point-of-care (e.g. doctors offices, examination rooms or census surveying locations). We use only the open-access data from the ISIC Archive, which comprises around 14k images labeled by professional dermatologists along with biopsy confirmation. Our results demonstrated that a smartphone application is able to embed a complete CNN inference model capable of assisting dermatologists in skin lesion examination, obtaining at least $90 \%$ accuracy for malignant melanoma detection.

\section{BACKGROUND}

In this section we review the fundamentals of dermatological diagnosis, a hierarchy of skin lesions, the evolution of image recognition networks and their practical usage.

\section{A. Dermatological Diagnosis}

Dermatoscopy is a procedure carried out by dermatologists, who visually examine skin lesions in order to detect anomalous features that might characterize a specific disease. This procedure uses an optical apparatus (a dermatoscope with lenses and camera) to obtain high-quality images of the skin, which are evaluated according to a set of criteria, such as the "ABCDE" criteria [10]. This acronym summarizes the most distinguishing features in the group of diseases called cutaneous melanoma, i.e. a cancerous growth of pigmented cells (melanocytes) in the skin. These features include:

- Asymmetry of the lesion shape, characterizing an accelerating growth of larger areas in certain directions; 
- Border irregularity, characterizing an accelerating growth in several small spots distributed over the lesion;

- Color variation, caused by necrosis, melanin production, changes in subcutaneous tissue, vascular damage, etc;

- Diameter $>6 \mathrm{~mm}$, for which a millimetric ruler is superimposed in the dermatoscopic image;

- Evolution of previous features over a certain timespan, e.g. a slow change in color over several months, which may not be observed by criterion $\mathrm{C}$ in a single image.

The combination of these features and their magnitude are analyzed by the dermatologist in order to assign a preliminar diagnostic to every lesion. If the diagnostic has sufficient support in the literature to pose a health hazard, such as the potential to spread to and impair the functioning of other organs, the lesion is said malignant, and the dermatologist proceeds to a costlier but more sensitive test, e.g. a biopsy.

\section{B. The International Skin Imaging Collaboration}

The International Skin Imaging Collaboration (ISIC) 2017 dataset provides 2.000 skin lesion images with masks for segmentation, superpixel masks for dermoscopic feature extraction, and annotations for classification. It is suitable for training machine learning algorithms. The lesions in the images classify into Melanoma, Seborrheic keratosis, and Nevus. Melanoma, the most critical, refers to a malignant skin tumor. The other two kinds are said to be benign skin tumors, derived cells that do not pose the threat of spreading across the body, but, that, still deserve treatment.

Fig. 1. Samples from the ISIC dataset. In the first row, the original images; in the second row, the segmentation masks. Reproduced from [11].

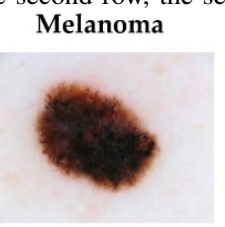
Seborrheic keratosis Nevus
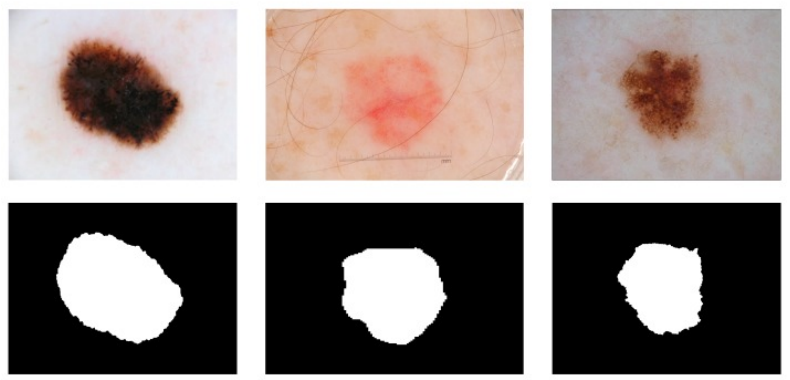

Figure 1 presents the samples from the ISIC dataset along with masks for different tasks. The first row presents the original skin lesion images; the second row shows the masks for segmentation. Besides this training bundle, the ISIC 2017 also provides a validation set with another 150 skin lesion images to evaluate the performance of the algorithms.

\section{The ILSVRC challenge}

The ILSVRC [12] is an annual competition for image recognition algorithms. The dataset used in the ILSVRC challenge contains more than a million pictures from several Internet sources, of which $50 \mathrm{k}$ pictures are randomly sampled and released before the competition for validation purposes, and another sample with $150 \mathrm{k}$ images is used for final scoring of the submissions. One of the challenges in the ILSVRC is to assign the top five (of a thousand) classes most similar for each object portrayed in a given image. In 2012, the AlexNet CNN [13] achieved an error rate of $15.3 \%$ in the top-5 challenge. This error rate granted to AlexNet the 2012's best result and also a breakthrough for image recognition with neural networks over large datasets. Afterwards, at least $95 \%$ of the ILSVRC submissions and $100 \%$ of the first places from 2013 to 2017 derived their algorithms from research on neural networks ${ }^{1}$ effectively presenting $\mathrm{CNNs}$ as a feasible and efficient method for large-scale image recognition. An increase in deep learning research was observed in other events, such as the 2017 editions of IEEE CVPR [14] and SIBGRAPI [15].

\section{Deep Learning from Images}

Deep learning (DL) is a computational method that learns representations of data with multiple levels of abstraction by using models that mimic the dynamics of a nervous system composed of neurons layers. DL introduces a number of processing, data, and mathematical innovations that have dramatically improved the state-of-the-art in speech recognition, visual recognition, object detection, object segmentation and many other domains such as drug discovery and genomics.

DL is capable of discovering intricate structures; to do so, it optimizes an object distribution in the vector space (objectified by the choice of model and loss function) by iteratively deriving clustering errors and gradually backpropagating the proportional error terms to the millions of parameters (weights) in between layers of neurons until a desired objective is reached, as measured by precision, accuracy and other scores in tasks of classification, recognition and segmentation. Two architectural variations of DL are of special interest; deep CNNs have brought about breakthroughs in processing images, video, speech and audio, whereas recurrent nets (RNNs) have shone light on sequential data such as text and speech [16].

The main feature of DL is its ability to automatically learn features from the input data; such features correspond to the details that numerically represent the details of a given data item. Previously to DL, the determination of the features was a task that had to be done manually by specialists with knowledge about the data domain. When performed manually, only a limited number of features could be defined, many times the features were not effective and were sensitive to variations of data, like linear transformations. With DL, the features become intrinsic to the model. Another advantage of DL is that it allows for transfer learning; that is, a model can trained and parametrized for a given data domain, say wheeled vehicles, and, later, be easily adjusted to a related data domain, like sports cars. All of these traits render DL to be a technology that organizes in the form of convenient software libraries and that advances throughout multiple domains iteratively or even in parallel. As a result, despite its advances and complexity, DL is a highly accessible technology whose advances heavily rely on the acquisition of structured, large, and labeled datasets,

\footnotetext{
${ }^{1}$ From: Challenges/ILSVRC/Results at http://image-net.org/ on 2018-06-14.
} 
and on computing resources powerful enough to process very large volumes of data in reasonable time.

\section{E. Model Optimization}

Mobile Optimization: Tensorflow-lite, MobileNet

\section{Methodology}

In this section we describe the methods used for data preprocessing, deep learning, and application design.

\section{A. Normalization}

- DPI adjustment

- Crop border occlusions

- Split in 32/64/128/256 square patches

- Select ROIs: patches with larger variance and infrequent pigments

- Brightness/Contrast adjusted by initial 1x1 convolutions

\section{B. Augmentation}

- Rotations

- Scaling

- Lighting

- Skin color

\section{Training}

- all ROIs are marked with the label

- transfer Inception-v3 learning to the skin domain

- adding residual links

- further training until stabilization

\section{Deployment and Classification}

- Mobile model is made available for download

- Normalize image (as defined in section III-A)

- Classify the obtained patches

- Show result as original image with overlaid labels

\section{E. Application User Interface}

- Medical staff may annotate (supervising) the result

- share their cases with other doctors

- help colleagues in their cases

- download different/updated models

\section{RELATED WORKS}

The work presented by Ruiz et al. [17] presents a clinical decision system that classifies images with suspicious lesions on the skin to assist as a second opinion clinical protocol. The authors combine the nearest K-neighbors method, a multi-layer Perceptron (MLP), and a parametric classifier based on Bayes' decision theory to achieve a collaborative classification system. They use a set of descriptors that comprises the variation of the sharpness, color homogeneity, mean of the R and $G$ components, and average of the HSL color luminosity. They obtained a precision of $87.76 \%$.

The work of Yu et al. [18] describes an automatic melanoma diagnosis system. The authors use a Convolutional Residual Network to segment the images during preprocessing. In a second step, they use Very Deep Residual Network for classification. For the training, they use 900 pre-labeled images and 350 images for testing; this method obtained an accuracy of $85.5 \%$.

The work presented by Souza et al. [19] uses decision trees based on descriptors of asymmetry, edge, and coloring to distinguish between images that present melanoma skin cancer from others that present common spots. The highest reported accuracy was of $90 \%$. The work presented by Aswin et al. [20] also works over the identification of melanoma using RNAs. The authors use a wide set of descriptors incluidng contrast, correlation, homogeneity, Second Moment, and RGB color variance. In this work, RNAs were used and the accuracy was $88 \%$.

Nasr-Esfahani et al. [21] describe an automatic melanoma diagnosis system that uses a preprocessing step to correct the illumination, to segment the images, and to enhance the images through a Gaussian filter. Next, they use a Convolutional Neural Network for classification, which also extracts the salient characteristics of the images. This method obtained an accuracy of $81 \%$. Majtner et al. [22] proposes a system that automatically classifies melanomas; in their system, the descriptor vector of each image is obtained using RSurf, Local Binary Patterns (LBP), and Convolutional Neural Networks. Furhter, they use the technique Support Vector Machines (SVM) for classification. During training, they used $900 \mathrm{im}-$ ages; for testing, they used 379 images. The highest accuracy reported by the authors was of $82,6 \%$.

Our work differs from previous ones as it achieves an accurary at the order of $90 \%$ inspired by the latest achievements regarding neural network archictecutes. It also innovates with a design dedicated to mobile platforms, which demanded processing load adjustments and specific interface choices.

\section{EXPERIMENTS}

In this section we exhibit the results achieved by our method in the ISIC dataset, discussing the effects of different settings and comparing to previous reports in the literature.

The software was implemented using Python-3.6, Inceptionv3, TensorFlow-1.13, CUDA-10 and CUDNN-7 libraries. All tests ran on a CentOS-7 system with an Intel-Core-i7-7700K processor (4-core/8-thread up to $4.5 \mathrm{GHz}$ ), 16G-DDR3 memory, an NVIDIA-Titan-Xp graphics card (3,840 cores up to $1.58 \mathrm{GHz}$ with $12 \mathrm{G}-\mathrm{G} 5 \mathrm{X}$ memory), a Gigabyte-H110M-H motherboard, and a 750GB Samsung-SpinPoint-F1-DT hard disk drive. The mobile application was implemented using Google-Android-Studio and Android-SDK tools. ${ }^{2}$

\section{A. Data preprocessing}

The preprocessing stage comprised operations of normalization of images (DPI, brightness, contrast, resolution), increase of the dataset cardinality (simulating color variations, illumination, deformations, and rotations), and image segmentation. The following operations were performed on the ISIC dataset:

- DPI and size adjustment to $1000 \times 1000$ resolution;

${ }^{2}$ All trademarks are copyrighted by their respective owners. 
- In conjunction with segmentation masks, the regions of interest were selected by the masks;

- The edges of the images were discarded;

- Histogram normalization: contrast, brightness, and gamma per channel (R, G, B), adjusting differences due to the use of different types of equipment.

Meanwhile, the operations of zoom, rotation, and resizing were performed online, each time the images were resubmitted to the video memory.

It is worth noting that some of these operations are also executed intrinsically in the neural network model; for example, the brightness and contrast are also adjusted by the initial $1 \times 1$ convolutions.

With the preprocessed data set, the images were separated into three sets: training (feedforward propagation), validation (estimation of the loss function and backpropagation), and test (evaluation of the final precision via cross-validation). In the literature, several values are used for the amount of data used in each step. Goodfellow et al. 23 suggests a 50\%, $25 \%$ and $25 \%$ split; which distributes an equal division of data between training and verification sets (test + validation).

\section{B. Training}

The training stage, when the weights of the network are adjusted so to perform the best image-to-class mapping, had the following steps:

- Assemble of the training images with their respective labels;

- Retrain of the last layers of the Inception-v3 neural network: the weights of the fully connected and softmax output layers were reset, and were submitted to successive iterations of backpropagation;

- Retrain of all the layers of Inception-v3: the nearest weights of the output were mixed with noise, and the backpropagation was performed throughout the entire network;

- Alteration of the in Inception-v3 structure: we added ResNet links for residual propagation to the Inception module. Such a change could be performed dynamically, followed by complete re-training with a smaller number of iterations.

After each training cycle, we evaluated the model for each class of skin lesion present in the data. To do so, the validation images (with and without normalization, separately) were classified by the network. Then, the classes of the validation images are compared to the output of the model, and a matrix of confusion is assembled. Finally, we used traditional evaluation measures, such as Precision-Recall, Accuracy, F1measure, and area under the ROC curve (sensitivity $\times$ specificity).

\section{CONCLUSION}

DermaDL is a complete solution to aid dermatological diagnosis at the cost of a common smartphone. It provides a mobile application which runs a lightened neural network to classify images obtained by dermatologists, helping them to retrieve related information from the patient history and compare them with similar cases in the literature while achieving results compatible with professional medical training.

This application has the potential to be a general pointof-care diagnosis support tool, by simply setting the correct model for each specific kind of examination, e.g., a CNN model trained to recognize small fractures in chest $x$-ray images, or a natural language model to digitize textual blood tests, an audio processing model to evaluate hearing damage, and so on. DermaDL is an initial platform for general inference models, thus fully extensible by on-demand updates.

Its architecture was designed to evolve in the cloud, creating a collaborative research network where multidisciplinary, multi-institutional practitioners and researchers can contribute with datasets and algorithms, given appropriate publishing standards, whose results can be easily validated and used by the clinical staff. As future work we plan to develop more models, connect this service with other dataset archives and collaborate with more medical researchers in the extension of the system.

\section{ACKNOWLEDGMENT}

This study was financed in part by the Coordenação de Aperfeiçoamento de Pessoal de Nível Superior - Brasil (CAPES) - Finance code 001 and grants $\mathrm{N}^{\circ}$.: PROEX7494731/D, PROEX-7494731/D1, PROEX-7494731/D2; by the São Paulo Research Foundation (FAPESP) grant $\mathrm{N}^{\circ}$.: 2016/17078-0; and the National Council for Scientific and Technological Development (CNPq) grants $\mathrm{N}^{\circ} .:$ 305580/20175 and 406550/2018-2. We also thank NVIDIA Corporation for the donation of a Titan Xp graphics card.

\section{REFERENCES}

[1] W. G. Hatcher and W. Yu, "A survey of deep learning: Platforms, applications and emerging research trends," IEEE Access, vol. 6, pp. 24 411-24 432, 2018.

[2] D. Schadendorf, A. C. J. van Akkooi, C. Berking, K. G. Griewank, R. Gutzmer, A. Hauschild, A. Stang, A. Roesch, and S. Ugurel, "Melanoma," The Lancet, vol. 392, no. 10151, pp. 971 - 984, 2018. [Online]. Available: https://doi.org/10.1016/S0140-6736(18)31559-9

[3] D. S. Rigel and J. A. Carucci, "Malignant melanoma: Prevention, early detection, and treatment in the 21st century," CA: A Cancer Journal for Clinicians, vol. 50, no. 4, pp. 215-236, 2000. [Online]. Available: https://doi.org/10.3322/canjclin.50.4.215

[4] T. Ching, D. S. Himmelstein, B. K. Beaulieu-Jones, A. A. Kalinin, B. T. Do, G. P. Way, E. Ferrero, P.-M. Agapow, M. Zietz, M. M. Hoffman, W. Xie, G. L. Rosen, B. J. Lengerich, J. Israeli, J. Lanchantin, S. Woloszynek, A. E. Carpenter, A. Shrikumar, J. Xu, E. M. Cofer, C. A. Lavender, S. C. Turaga, A. M. Alexandari, Z. Lu, D. J. Harris, D. DeCaprio, Y. Qi, A. Kundaje, Y. Peng, L. K. Wiley, M. H. S. Segler, S. M. Boca, S. J. Swamidass, A. Huang, A. Gitter, and C. S. Greene, "Opportunities and obstacles for deep learning in biology and medicine," Journal of The Royal Society Interface, vol. 15, no. 141, 2018. [Online]. Available: http://rsif.royalsocietypublishing.org/content/15/141/20170387

[5] V. U. Sameer, R. Naskar, N. Musthyala, and K. Kokkalla, "Deep learning based counter-forensic image classification for camera model identification," in Digital Forensics and Watermarking, C. Kraetzer, Y.Q. Shi, J. Dittmann, and H. J. Kim, Eds. Cham: Springer International Publishing, 2017, pp. 52-64. 
[6] R. Rocha, A. Siravenha, A. C. S. Gomes, G. L. Serejo, A. F. B. Silva, L. Mousinho Rodrigues, J. Braga, G. Dias, and S. Carvalho, "Avaliação de técnicas de deep learning aplicadas à identificação de peças defeituosas em vagões de trem," in Workshop of Industry Applications (WIA) of the 30th Conference on Graphics, Patterns and Images (SIBGRAPI'17), Oct 2017.

[7] A. Kamilaris and F. X. Prenafeta-Boldu, "Deep learning in agriculture: A survey," Computers and Electronics in Agriculture, vol. 147, pp. 70-90, 2018. [Online]. Available: http://www.sciencedirect.com/science/ article/pii/S0168169917308803

[8] A. Esteva, B. Kuprel, R. A. Novoa, J. Ko, S. M. Swetter, H. M. Blau, and S. Thrun, "Dermatologist-level classification of skin cancer with deep neural networks," Nature, vol. 542, pp. 115-118, 2017.

[9] C. Szegedy, V. Vanhoucke, S. Ioffe, J. Shlens, and Z. Wojna, "Rethinking the inception architecture for computer vision," in The IEEE Conference on Computer Vision and Pattern Recognition (CVPR), June 2016.

[10] A. NR, S. HM, R. DS, and et al, "Early diagnosis of cutaneous melanoma: Revisiting the ABCD criteria," JAMA, vol. 292, no. 22, pp. 2771-2776, 2004. [Online]. Available: http://dx.doi.org/10.1001/jama. 292.22.2771

[11] Y. Li and L. Shen, "Skin lesion analysis towards melanoma detection using deep learning network," Sensors (Basel), vol. 18, no. 2, p. 556, Feb 2018. [Online]. Available: http://www.ncbi.nlm.nih.gov/pmc/ articles/PMC5855504/

[12] O. Russakovsky, J. Deng, H. Su, J. Krause, S. Satheesh, S. Ma Z. Huang, A. Karpathy, A. Khosla, M. Bernstein, A. C. Berg, and L. Fei-Fei, "ImageNet Large Scale Visual Recognition Challenge," International Journal of Computer Vision (IJCV), vol. 115, no. 3, pp. 211-252, 2015.

[13] A. Krizhevsky, I. Sutskever, and G. E. Hinton, "Imagenet classification with deep convolutional neural networks," in Advances in Neural Information Processing Systems 25, F. Pereira, C. J. C. Burges, L. Bottou, and K. Q. Weinberger, Eds. Curran Associates, Inc., 2012, pp. 1097-1105. [Online]. Available: http://papers.nips.cc/paper/ 4824-imagenet-classification-with-deep-convolutional-neural-networks. pdf

[14] "Table of contents," in 2017 IEEE Conference on Computer Vision and Pattern Recognition (CVPR), July 2017, pp. v-liii.

[15] "Table of contents," in 2017 30th SIBGRAPI Conference on Graphics, Patterns and Images (SIBGRAPI), Oct 2017, pp. v-x.

[16] Y. LeCun, Y. Bengio, and G. Hinton, "Deep learning," Nature, vol 521, p. 436 EP, May 2015. [Online]. Available: http://dx.doi.org/10. 1038/nature14539

[17] D. Ruiz, V. Berenguer, A. Soriano, and B. Sánchez, "A decision support system for the diagnosis of melanoma: A comparative approach," Expert Systems with Applications, vol. 38, no. 12, pp. 15217-15223, 2011. [Online]. Available: http://www.sciencedirect.com/science/article/ pii/S0957417411008633

[18] L. Yu, H. Chen, Q. Dou, J. Qin, and P. A. Heng, "Automated melanoma recognition in dermoscopy images via very deep residual networks," IEEE Transactions on Medical Imaging, vol. 36, no. 4, pp. 994-1004, 2017.

[19] H. de Souza Ganzeli, J. G. Bottesini, L. de Oliveira Paz, and M. F. S. Ribeiro, "Skan: Skin scanner - system for skin cancer detection using adaptive techniques," IEEE Latin America Transactions, vol. 9, no. 2, pp. 206-212, 2011.

[20] R. B. Aswin, J. A. Jaleel, and S. Salim, "Hybrid genetic algorithm artificial neural network classifier for skin cancer detection," in 2014 International Conference on Control, Instrumentation, Communication and Computational Technologies (ICCICCT), July 2014, pp. 1304-1309.

[21] E. Nasr-Esfahani, S. Samavi, N. Karimi, S. M. R. Soroushmehr, M. H Jafari, K. Ward, and K. Najarian, "Melanoma detection by analysis of clinical images using convolutional neural network," in 2016 38th Annual International Conference of the IEEE Engineering in Medicine and Biology Society (EMBC), 2016, pp. 1373-1376.

[22] T. Majtner, S. Yildirim-Yayilgan, and J. Y. Hardeberg, "Combining deep learning and hand-crafted features for skin lesion classification," in 2016 Sixth International Conference on Image Processing Theory, Tools and Applications (IPTA), 2016, pp. 1-6.

[23] I. Goodfellow, Y. Bengio, A. Courville, and Y. Bengio, Deep learning. MIT press Cambridge, 2016, vol. 1. 